\title{
Synthesis of Chiral Pyrimidin-2(1H)-ones from $N$-Carbamoyl Amino Acids
}

\author{
Ilya N. Egorov ${ }^{\mathrm{a}}$, Vladimir L. Rusinov ${ }^{\mathrm{a}, \mathrm{b}}$ and Oleg N. Chupakhin ${ }^{\mathrm{a}, \mathrm{b}}$ \\ a Institute of Chemistry and Technology, Ural Federal University, Mira St. 19, Ekaterinburg, \\ 620 002, Russia \\ b Institute of Organic Synthesis of Russian Academy of Sciences, S. Kovalevskoy St. 22, \\ Ekaterinburg, 620 041, Russia \\ Reprint requests to Dr. Ilya Egorov. Fax: +7(343)3740458. E-mail: i.n.egorov@ gmail.com
}

Z. Naturforsch. 2013, 68b, 1253 - 1258 / DOI: 10.5560/ZNB.2013-3129

Received May 1, 2013

A series of previously unknown pyrimidin-2(1H)-ones containing chiral amino acid fragments was synthesized from 1,1,3,3-tetramethoxypropane and $N$-carbamoyl derivatives of amino acids under acidic conditions.

Key words: Heterocycles, Amino Acids, Condensation, Chirality, Pyrimidines

\section{Introduction}

Amino acid derivatives of pyrimidinones are nucleoside analogs and are interesting as biologically active compounds or their precursors [1]. It is known that pyrimidin-2(1H)-ones 1 containing peptide fragments reveal anti-inflammatory activity [2]. Their aza analogs - 1.2.4-triazin-3(2H)-ones 2 - do not show or show only low anti-inflammatory activity, however these compounds have been proved to show strong analgesic properties [3]. Pyrimidinones $\mathbf{3}$ are useful in the treatment or prevention of the diseases mediated by the activation of the $\beta 3$-adrenoceptor $[4,5]$.

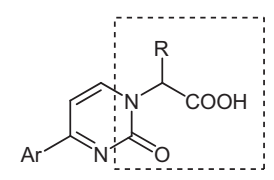

1

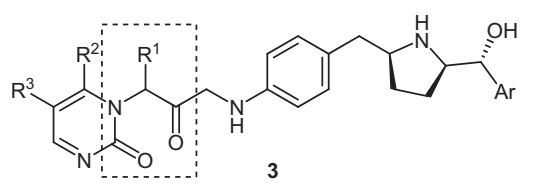

The published methods [1-4] for the synthesis of compounds 1-3 lead to products with racemic amino acid fragments, but it is known that chirality is important for bioactivity. To the best of our knowledge only few methods of synthesis of chiral $\alpha$-amino acid derivatives of pyrimidines are known [6-8]. One method of synthesis of pyrimidines with chiral $\alpha$ amino acid fragments at the heterocyclic ring has been reported. The alkylation of cytosine with esters of $(S)$-lactic acid leads to alkyl derivatives of cytosine $(d e=56)[8]$.

\section{Results and Discussion}

In this work the synthesis of pyrimidin-2(1H)ones with amino acid residues is described. It is known that $N$-carbamoyl amino acids easily cyclize to give hydantoins under acidic conditions $[9,10]$. The condensation of 1,1,3,3-tetramethoxypropane with $\mathrm{N}$ carbamoyl derivatives of amino acids in the presence of hydrochloric acid in methanol gives methyl esters of pyrimidinones 5-11 (Scheme 1, Fig. 1). The change of solvent from methanol to ethanol, 2-propanol or water leads to the disappearance of pyrimidinones in the products of the reaction. In the reactions with $N$ carbamoyl derivatives of other amino acids such as Lvaline, L-leucine, L-isoleucine, D, L-tryptophan, or Lserine we failed to obtain the corresponding pyrimidinones. Acid hydrolysis of the esters $\mathbf{5}$ and $\mathbf{6}$ leads to carboxylic acids $\mathbf{1 2}(67 \%)$ and $\mathbf{1 3}(76 \%)$, respectively (Scheme 1). 
<smiles>COC(CC(OC)OC)OC</smiles>

4<smiles>[R]C(NC(N)=O)C(=O)O</smiles><smiles>COCCOCCO</smiles>
r. t.<smiles>[R]C(C)(C)n1cccnc1=O</smiles>

5-11<smiles>C[C@@H](C(=O)O)n1cccnc1=O</smiles>

12,13

\begin{tabular}{llll}
\hline $\mathrm{R}$ & Configuration & Product & Yield (\%) \\
\hline $\mathrm{CH}_{3}$ & $(S)$ & $\mathbf{5}$ & 43 \\
$\mathrm{CH}_{3}$ & $(R)$ & $\mathbf{6}$ & 21 \\
$\mathrm{CH}_{2} \mathrm{CH}_{2} \mathrm{SCH}_{3}$ & $(S)$ & 7 & 55 \\
$\mathrm{CH}_{2} \mathrm{CH}_{2} \mathrm{SCH}_{3}$ & $(R)$ & $\mathbf{8}$ & 15 \\
$\mathrm{CH}_{2} \mathrm{CH}_{2} \mathrm{SCH}_{3}$ & $(R, S)$ & $\mathbf{9}$ & 34 \\
$\mathrm{CH}_{2} \mathrm{Ph}$ & $(R, S)$ & $\mathbf{1 0}$ & 40 \\
$\mathrm{CH}_{2}\left(p-\mathrm{C}_{6} \mathrm{H}_{4} \mathrm{OH}\right)$ & $(S)$ & $\mathbf{1 1}$ & 52 \\
& & & \\
\hline
\end{tabular}

Scheme 1. Synthesis of pyrimidin-2(1H)-one derivatives.

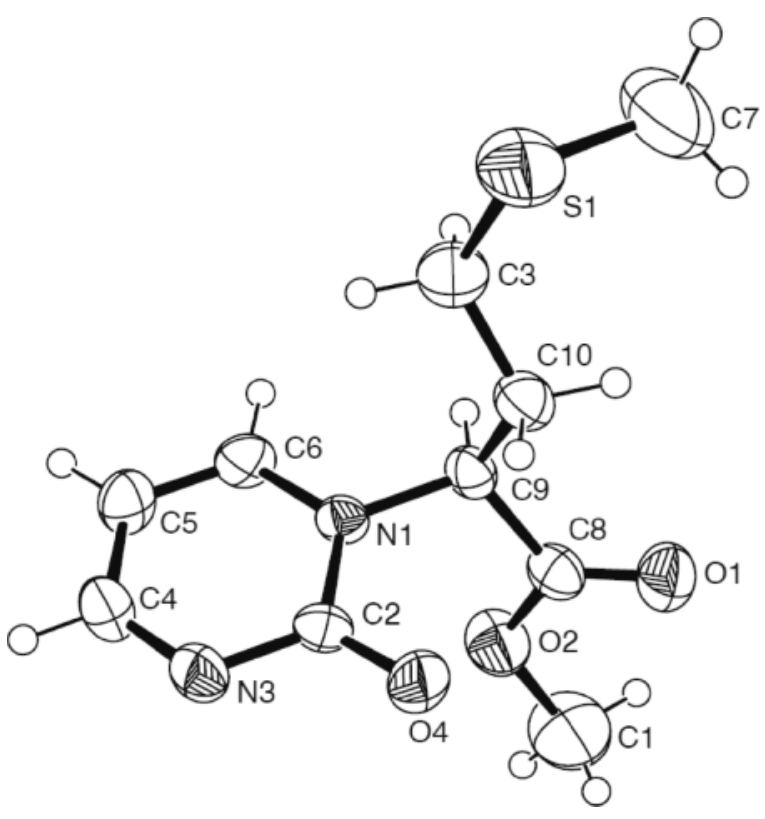

Fig. 1. ORTEP diagram of 7.
In addition we tried to prepare 1-substituted pyrimidin-2-ones from 2-phenylmalonaldehyde 14. When a mixture of 2-phenylmalonaldehyde and a substituted urea was refluxed in toluene in the presence of $p$-TsOH [11] hydantoins were formed as the sole products from the $\mathrm{N}$-carbamoyl derivatives of $\mathrm{L}$ methionine, L-lysine, L-leucine, L-isoleucine, and Ltyrosine. Using different reaction times in the reaction with L-valine led to the products $\mathbf{1 5}$ and $\mathbf{1 6}$ besides the corresponding hydantoin derivative (Scheme 2).

Obviously the first step of the reaction leads to the condensation product 15 (Fig. 2). Under forced conditions $\mathbf{1 5}$ is cyclized and subsequently decarboxylated to give $\mathbf{1 6}$ (Fig. 3). We could not find conditions which allowed the isolation of an intermediate. When benzene or $p$-xylene were used as solvents, hydantoins were the sole reaction products.

\section{Experimental Section}

All commercially obtained solvents and reagents were used as received; $N$-carbamoyl amino acids [12] and 2- 


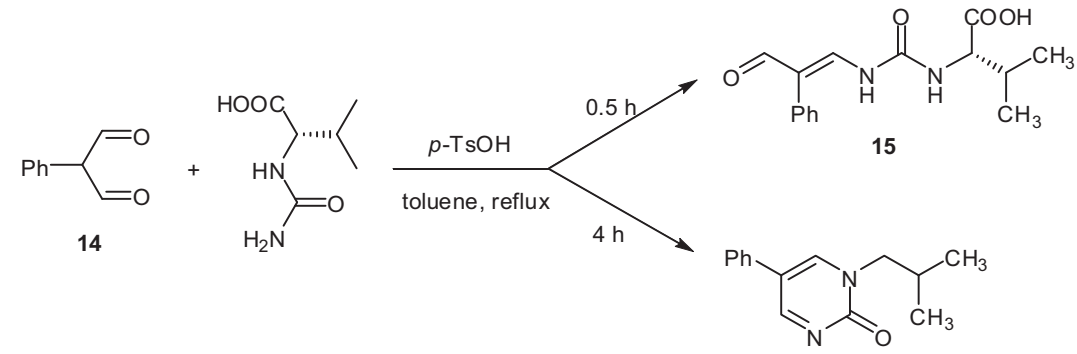

16

Scheme 2.

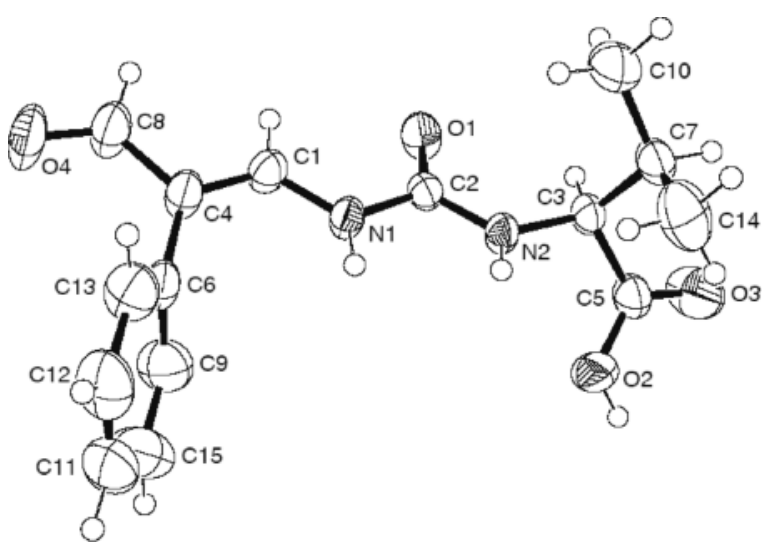

Fig. 2. ORTEP diagram of $\mathbf{1 5 .}$

phenylmalonaldehyde [11] were synthesized by known methods. ${ }^{1} \mathrm{H},{ }^{13} \mathrm{C}$ NMR spectra were recorded on a Bruker DRX-400 spectrometer; tetramethylsilane (TMS) was used as an internal standard. The optical rotation was measured on a Perkin Elmer polarimeter. Mass spectra were recorded on a Bruker Daltonics MicroTOF-Q II mass spectrometer with electrospray ionization.

General procedure for the synthesis of compounds 5-11

1,1,3,3-Tetramethoxypropane $(821 \mu \mathrm{L}, 5.0 \mathrm{mmol})$ and conc. $\mathrm{HCl}(12 \mathrm{M}, 3 \mathrm{~mL})$ were added to a suspension of the corresponding $N$-carbamoyl amino acid $(5.0 \mathrm{mmol})$ in methanol $(10 \mathrm{~mL})$. After a few minutes a colorless suspension resulted, which turned to a clear yellow solution on stirring at ambient temperature for 1 day. Then it was neutralized with saturated aqueous $\mathrm{NaHCO}_{3}$. After evaporation of methanol the solution was diluted with $40 \mathrm{~mL}$ of water and extracted three times with $\mathrm{CHCl}_{3}(50 \mathrm{~mL})$. The organic layer was separated, washed with water and brine and dried over $\mathrm{Na}_{2} \mathrm{SO}_{4}$. The $\mathrm{CHCl}_{3}$ was evaporated, and the residue was chromatographed on a silica gel column with EtOAc as an eluent (5-9). Compounds 10, 11 were crystallized from ethanol.

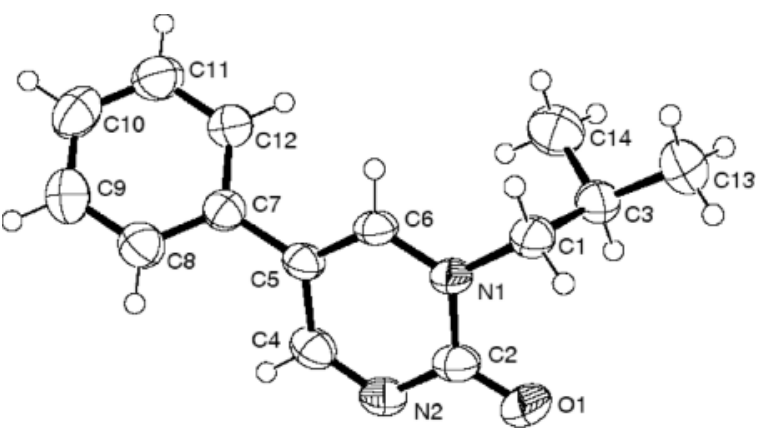

Fig. 3. ORTEP diagram of $\mathbf{1 6 .}$

(S)-2-(2-Oxopyrimidin-1(2H)-yl)propionic acid methyl ether (5)

Colorless liquid. Yield $0.39 \mathrm{~g}(43 \%) . \quad-{ }^{1} \mathrm{H}$ NMR (400 MHz, [D $\left.\left.\mathrm{D}_{6}\right] \mathrm{DMSO}\right): \delta=1.67\left(\mathrm{~d}, J=7.4 \mathrm{~Hz}, 3 \mathrm{H}, \mathrm{CH}_{3}\right.$ ), $3.76\left(\mathrm{~s}, 3 \mathrm{H}, \mathrm{OCH}_{3}\right), 5.41$ (q, $\left.J=7.4 \mathrm{~Hz}, 1 \mathrm{H}, \mathrm{CH}\right), 6.38(\mathrm{dd}$, $J=6.7,4.1 \mathrm{~Hz}, 1 \mathrm{H}, \mathrm{Py}-\mathrm{H}), 7.74(\mathrm{dd}, J=6.7,2.8 \mathrm{~Hz}, 1 \mathrm{H}$, Py-H), 8.60 (dd, $J=3.9,2.9 \mathrm{~Hz}, 1 \mathrm{H}, \mathrm{Py}-\mathrm{H}) .-{ }^{13} \mathrm{C}$ NMR (100 MHz, [D 6 DMSO) $\delta=16.5,53.0,55.6,104.2,145.4$, 155.7, 166.1, 170.4. $-\mathrm{C}_{8} \mathrm{H}_{10} \mathrm{~N}_{2} \mathrm{O}_{3}$ : calcd. C 52.74, H 5.53, N 15.38; found C 52.99, H 5.67, N, 15.45.

(R)-2-(2-Oxopyrimidin-1(2H)-yl)propionic acid methyl ester (6)

Colorless liquid. Yield $0.19 \mathrm{~g}(21 \%) . \quad-{ }^{1} \mathrm{H}$ NMR (400 MHz, [D $\left.\left.{ }_{6}\right] \mathrm{DMSO}\right) \delta=1.67\left(\mathrm{~d}, J=7.4 \mathrm{~Hz}, 3 \mathrm{H}, \mathrm{CH}_{3}\right)$, $3.76\left(\mathrm{~s}, 3 \mathrm{H}, \mathrm{OCH}_{3}\right), 5.41$ (q, $\left.J=7.4 \mathrm{~Hz}, 1 \mathrm{H}, \mathrm{CH}\right), 6.38(\mathrm{dd}$, $J=6.7,4.1 \mathrm{~Hz}, 1 \mathrm{H}, \mathrm{Py}-\mathrm{H}), 7.74(\mathrm{dd}, J=6.7,2.8 \mathrm{~Hz}, 1 \mathrm{H}$, Py-H), 8.60 (dd, $J=3.9,2.9 \mathrm{~Hz}, 1 \mathrm{H}, \mathrm{Py}-\mathrm{H}) .-\mathrm{C}_{8} \mathrm{H}_{10} \mathrm{~N}_{2} \mathrm{O}_{3}$ : calcd. C 52.74, H 5.53, N 15.38; found C 52.82, H 5.70, N 15.27 .

(S)-4-(Methylthio)-2-(2-oxopyrimidin-1(2H)-yl)butyric acid methyl ester (7)

Colorless crystals. Yield $0.67 \mathrm{~g}(55 \%)$; m.p. $129^{\circ} \mathrm{C}$. $[\alpha]_{\mathrm{D}}=-100.8(c=1.0, \mathrm{MeOH}) .-{ }^{1} \mathrm{H} \mathrm{NMR}(400 \mathrm{MHz}$, [D $\mathrm{D}_{6}$ DMSO): $\delta=2.01$ (s, 3H, $\left.\mathrm{SCH}_{3}\right), 2.32-2.44(\mathrm{~m}, 4 \mathrm{H}$, 


\begin{tabular}{|c|c|c|c|c|}
\hline & 7 & 15 & 16 & Table 1. Crystal structure data \\
\hline Empirical formula & $\mathrm{C}_{10} \mathrm{H}_{14} \mathrm{~N}_{2} \mathrm{O}_{3} \mathrm{~S}$ & $\mathrm{C}_{15} \mathrm{H}_{18} \mathrm{~N}_{2} \mathrm{O}_{4}$ & $\mathrm{C}_{14} \mathrm{H}_{16} \mathrm{~N}_{2} \mathrm{O}$ & \\
\hline$M_{\mathrm{r}}$ & 242.29 & 290.31 & 228.29 & \\
\hline Crystal size, $\mathrm{mm}^{3}$ & $0.25 \times 0.13 \times 0.07$ & $0.24 \times 0.13 \times 0.08$ & $0.44 \times 0.21 \times 0.07$ & \\
\hline Crystal system & orthorhombic & monoclinic & triclinic & \\
\hline Space group & $P 2_{1} 2_{1} 2_{1}$ & $P 2_{1}$ & $P \overline{1}$ & \\
\hline$a, \AA$ & $6.1061(3)$ & $6.5984(3)$ & $6.2046(12)$ & \\
\hline$b, \AA$ & $13.8993(13)$ & $11.9696(5)$ & $9.368(3)$ & \\
\hline$c, \AA$ & $14.5862(10)$ & $9.9658(4)$ & $11.194(2)$ & \\
\hline$\alpha, \operatorname{deg}$ & 90.0 & 90.0 & $93.098(19)$ & \\
\hline$\beta, \operatorname{deg}$ & 90.0 & $93.980(3)$ & $103.775(17)$ & \\
\hline$\gamma$, deg & 90.0 & 90.0 & $102.96(2)$ & \\
\hline$V, \AA^{3}$ & $1237.94(16)$ & $785.20(6)$ & $611.9(2)$ & \\
\hline$Z$ & 4 & 2 & 2 & \\
\hline$D_{\text {calcd. }}, \mathrm{g} \mathrm{cm}^{-3}$ & 1.30 & 1.23 & 1.24 & \\
\hline$\mu\left(\operatorname{Mo} K_{\alpha}\right), \mathrm{cm}^{-1}$ & 0.3 & 0.1 & 0.1 & \\
\hline$F(000), \mathrm{e}$ & 512 & 308 & 244 & \\
\hline$h k l$ range & $+8 \rightarrow-6, \pm 19, \pm 20$ & $\pm 8, \pm 14,+12 \rightarrow-11$ & $+7 \rightarrow-6, \pm 11, \pm 13$ & \\
\hline$\theta$ range, deg & $2.79-30.52$ & $2.66-26.36$ & $2.76-26.37$ & \\
\hline Refl. measured/unique & $3691 / 2554$ & $1669 / 1283$ & $2481 / 1184$ & \\
\hline$R_{\text {int }}$ & 0.0165 & 0.0161 & 0.0236 & \\
\hline Param. refined & 147 & 206 & 154 & \\
\hline$R(F) / w R\left(F^{2}\right)^{\mathrm{a}}$ (all refl.) & $0.0575 / 0.0954$ & $0.0368 / 0.0557$ & $0.0870 / 0.0666$ & \\
\hline $\operatorname{GoF}\left(F^{2}\right)^{\mathrm{b}}$ & 1.002 & 1.006 & 1.007 & \\
\hline$\Delta \rho_{\text {fin }}(\max / \min )$, e $\AA^{-3}$ & $-0.25 / 0.22$ & $-0.11 / 0.09$ & $-0.13 / 0.10$ & \\
\hline
\end{tabular}

$\left.\mathrm{CH}_{2}\right), 3.65\left(\mathrm{~s}, 3 \mathrm{H}, \mathrm{OCH}_{3}\right), 5.12(\mathrm{t}, J=6.9 \mathrm{~Hz}, 1 \mathrm{H}, \mathrm{CH}), 6.51$ $(\mathrm{dd}, J=6.5,4.1 \mathrm{~Hz}, 1 \mathrm{H}, \mathrm{Py}-\mathrm{H}), 8.22(\mathrm{dd}, J=6.5,2.8 \mathrm{~Hz}$, $1 \mathrm{H}, \mathrm{Py}-\mathrm{H}), 8.60$ (dd, $J=4.1,2.8 \mathrm{~Hz}, 1 \mathrm{H}, \mathrm{Py}-\mathrm{H}) .-{ }^{13} \mathrm{C}$ NMR $\left(100 \mathrm{MHz},\left[\mathrm{D}_{6}\right] \mathrm{DMSO}\right): \delta=14.9,28.0,29.8,53.0,61.9$, 104.7, 149.9, 155.6, 167.3, 169.7. $-\mathrm{C}_{10} \mathrm{H}_{14} \mathrm{~N}_{2} \mathrm{O}_{3} \mathrm{~S}$ : calcd. C 49.57, H 5.82, N 11.56; found C 49.52, H 5.88, N 11.89 .

(R)-4-(Methylthio)-2-(2-oxopyrimidin-1(2H)-yl)butyric acid methyl ester (8)

Colorless crystals. Yield 0.18 g $(15 \%)$; m.p. $133^{\circ} \mathrm{C}$. $[\alpha]_{\mathrm{D}}=+94.3(c=1.0, \mathrm{MeOH}) .-{ }^{1} \mathrm{H}$ NMR $(400 \mathrm{MHz}$, [D $\mathrm{D}_{6}$ DMSO): $\delta=2.04\left(\mathrm{~s}, 3 \mathrm{H}, \mathrm{SCH}_{3}\right), 2.29-2.44(\mathrm{~m}, 4 \mathrm{H}$, $\left.\mathrm{CH}_{2}\right), 3.69\left(\mathrm{~s}, 3 \mathrm{H}, \mathrm{OCH}_{3}\right), 5.05-5.08(\mathrm{~m}, 1 \mathrm{H}, \mathrm{CH}), 6.43(\mathrm{dd}$, $J=6.5,4.1 \mathrm{~Hz}, 1 \mathrm{H}, \mathrm{Py}-\mathrm{H}), 8.15(\mathrm{dd}, J=6.5,2.8 \mathrm{~Hz}, 1 \mathrm{H}, \mathrm{Py}-$ $\mathrm{H}), 8.55(\mathrm{dd}, J=4.0,2.8 \mathrm{~Hz}, 1 \mathrm{H}, \mathrm{Py}-\mathrm{H}) .-\mathrm{C}_{10} \mathrm{H}_{14} \mathrm{~N}_{2} \mathrm{O}_{3} \mathrm{~S}$ : calcd. C 49.57, H 5.82, N 11.56; found C 49.64, H 5.93, N 11.70 .

(R,S)-4-(Methylthio)-2-(2-oxopyrimidin-1(2H)-yl)butyric acid methyl ester $(\mathbf{9})$

Colorless crystals. Yield 0.41 g $(34 \%)$; m.p. $105^{\circ} \mathrm{C}$. ${ }^{1} \mathrm{H}$ NMR (400 MHz, [D 6 ]DMSO): $\delta=2.04$ (s, 3H, $\mathrm{SCH}_{3}$ ), $2.32-2.43\left(\mathrm{~m}, 4 \mathrm{H}, \mathrm{CH}_{2}\right), 3.69\left(\mathrm{~s}, 3 \mathrm{H}, \mathrm{OCH}_{3}\right), 4.97-5.16$ $(\mathrm{m}, 1 \mathrm{H}, \mathrm{CH}), 6.43(\mathrm{dd}, J=6.5,4.1 \mathrm{~Hz}, \mathrm{Py}-\mathrm{H}), 8.15(\mathrm{dd}, J=$ $6.5,2.8 \mathrm{~Hz}, \mathrm{Py}-\mathrm{H}), 8.56(\mathrm{dd}, J=4.1,2.8 \mathrm{~Hz}, \mathrm{Py}-\mathrm{H}) .-{ }^{13} \mathrm{C}$ NMR (100 MHz, [D 6 DMSO): $\delta=14.9,28.1,29.9,53.0$, $61.9,104.6,149.8,155.6,167.3,169.7$. - HRMS $((+)$-ESI-
MS): $m / z=243.0848$ (calcd. 243.0798 for $\mathrm{C}_{10} \mathrm{H}_{15} \mathrm{~N}_{2} \mathrm{O}_{3} \mathrm{~S}$, $\left.[\mathrm{M}+\mathrm{H}]^{+}\right) .-\mathrm{C}_{10} \mathrm{H}_{14} \mathrm{~N}_{2} \mathrm{O}_{3} \mathrm{~S}$ : calcd. C 49.57, H 5.82, N 11.56; found C 49.54, H 5.86, N 11.60.

(R,S)-2-(2-Oxopyrimidin-1(2H)-yl)-3-phenylpropionic acid methyl ester (10)

Colorless crystals. Yield $0.52 \mathrm{~g}(40 \%)$; m.p. $162^{\circ} \mathrm{C}$. $-[\alpha]_{\mathrm{D}}=0(c=1.0, \mathrm{MeOH}) .-{ }^{1} \mathrm{H}$ NMR $(400 \mathrm{MHz}$, [D 6 ]DMSO): $\delta=3.36-3.46\left(\mathrm{~m}, 2 \mathrm{H}, \mathrm{CH}_{2}\right), 3.71(\mathrm{~s}, 3 \mathrm{H}$, $\left.\mathrm{OCH}_{3}\right), 5.19(\mathrm{dd}, J=10.1,5.7 \mathrm{~Hz}, 1 \mathrm{H}), 6.16(\mathrm{dd}, J=6.5$, $4.1 \mathrm{~Hz}, 1 \mathrm{H}), 7.04-7.11(\mathrm{~m}, 2 \mathrm{H}), 7.12-7.21(\mathrm{~m}, 3 \mathrm{H}), 7.74$ (dd, $J=6.5,2.8 \mathrm{~Hz}, 1 \mathrm{H}), 8.42$ (dd, $J=4.1,2.8 \mathrm{~Hz}, 1 \mathrm{H})$. $-{ }^{13} \mathrm{C}$ NMR (100 MHz, [D 6 ]DMSO): $\delta=33.5,52.4,64.0$, 103.5, 126.7, 128.4, 128.9, 136.1, 149.5, 154.9, 166.6, 168.7. - HRMS ((+)-ESI-MS): $m / z=259.1094$ (calcd. 259.1077 for $\left.\mathrm{C}_{14} \mathrm{H}_{15} \mathrm{~N}_{2} \mathrm{O}_{3},[\mathrm{M}+\mathrm{H}]^{+}\right)$.

(S)-3-(4-Hydroxyphenyl)-2-(2-oxopyrimidin-

$1(2 \mathrm{H})$-yl)propionic acid methyl ester (11)

Colorless crystals. Yield 0.71 g (52\%); m.p. $166^{\circ} \mathrm{C}$. $[\alpha]_{\mathrm{D}}=-242.9(c=1.0, \mathrm{MeOH}) .-{ }^{1} \mathrm{H}$ NMR $(400 \mathrm{MHz}$, [D 6 ]DMSO): $\delta=3.22-3.33(\mathrm{~m}, 2 \mathrm{H}), 3.67\left(\mathrm{~s}, 3 \mathrm{H}, \mathrm{OCH}_{3}\right)$, $5.16(\mathrm{dd}, J=10.2,5.6 \mathrm{~Hz}), 6.28(\mathrm{dd}, J=6.5,4.2 \mathrm{~Hz}), 6.59$ $(\mathrm{d}, J=8.3 \mathrm{~Hz}, 2 \mathrm{H}, 4-\mathrm{OH}-P h), 6.85(\mathrm{~d}, J=8.3 \mathrm{~Hz}, 2 \mathrm{H}$, 4-OH-Ph), 7.81 (dd, $J=6.5,2.7 \mathrm{~Hz}, 1 \mathrm{H}), 8.47-8.49(\mathrm{~m}$, $1 \mathrm{H}), 9.22(\mathrm{~s}, 1 \mathrm{H}) .-{ }^{13} \mathrm{C}$ NMR $\left(100 \mathrm{MHz},\left[\mathrm{D}_{6}\right] \mathrm{DMSO}\right)$ : $\delta=32.7,52.3,64.1,103.5,115.2,125.9,129.8,149.5$, 
154.9, 156.0, 166.5, 168.8. - HRMS ((+)-ESI-MS): $m / z=$ 275.1044 (calcd. 275.1026 for $\mathrm{C}_{14} \mathrm{H}_{15} \mathrm{~N}_{2} \mathrm{O}_{4},[\mathrm{M}+\mathrm{H}]^{+}$).

Procedure for the synthesis of 12 and 13

Conc. $\mathrm{HCl}(3 \mathrm{~mL})$ was added to the solution of pyrimidinone 5 or $6(200 \mu \mathrm{g})$ in dioxane $(10 \mathrm{~mL})$. The reaction mixture was stirred for 1 day, and then it was evaporated in a Petri dish to dryness and crystallized from 2-propanol.

\section{(S)-2-(2-Oxopyrimidin-1(2H)-yl)propionic acid (12)}

Colorless crystals. Yield $0.12 \mathrm{~g}(67 \%)$; m.p. $179{ }^{\circ} \mathrm{C}$. $[\alpha]_{\mathrm{D}}=-39.4(c=1.0, \mathrm{MeOH}) .-{ }^{1} \mathrm{H} \mathrm{NMR}(400 \mathrm{MHz}$, [D 6 ]DMSO): $\delta=1.66\left(\mathrm{~d}, J=7.3 \mathrm{~Hz}, 3 \mathrm{H}, \mathrm{CH}_{3}\right), 5.21(\mathrm{q}, J=$ $7.2 \mathrm{~Hz}, 1 \mathrm{H}), 6.72(\mathrm{dd}, J=6.4,5.0 \mathrm{~Hz}, 1 \mathrm{H}), 8.63-8.70(\mathrm{~m}$, $1 \mathrm{H}), 8.73$ (dd, $J=4.7,2.6 \mathrm{~Hz}, 1 \mathrm{H}) .-{ }^{13} \mathrm{C}$ NMR $(100 \mathrm{MHz}$, [D ${ }_{6}$ DMSO): $\delta=15.4,59.0,104.7,150.5,156.0,162.3$, 170.6. - HRMS ((+)-ESI-MS): $m / z=169.0616$ (calcd. 169.0608 for $\left.\mathrm{C}_{7} \mathrm{H}_{9} \mathrm{~N}_{2} \mathrm{O}_{3},[\mathrm{M}+\mathrm{H}]^{+}\right)$. $-\mathrm{C}_{7} \mathrm{H}_{8} \mathrm{~N}_{2} \mathrm{O}_{3}$ : calcd. C 50.00, H 4.80, N 16.66; found C 50.12, H 4.93, N 16.77 .

\section{(R)-2-(2-Oxopyrimidin-1(2H)-yl)propionic acid (13)}

Colorless crystals. Yield $0.14 \mathrm{~g} \quad(76 \%) ; \quad$ m.p. $183-184{ }^{\circ} \mathrm{C} .-[\alpha]_{\mathrm{D}}=+37.1(c=1.0, \mathrm{MeOH}) .-{ }^{1} \mathrm{H}$ NMR (400 MHz, [D 6 DMSO): $\delta=1.65(\mathrm{~d}, J=7.3 \mathrm{~Hz}$, $\left.3 \mathrm{H}, \mathrm{CH}_{3}\right), 5.20(\mathrm{q}, J=7.3 \mathrm{~Hz}, 1 \mathrm{H}, \mathrm{CH}), 6.70(\mathrm{dd}, J=6.3$, $5.0 \mathrm{~Hz}, 1 \mathrm{H}, \mathrm{Py}-\mathrm{H}), 8.66(\mathrm{dd}, J=6.4,2.5 \mathrm{~Hz}, 1 \mathrm{H}, \mathrm{Py}-\mathrm{H})$, 8.72 (dd, $J=4.8,2.6 \mathrm{~Hz}, 1 \mathrm{H}, \mathrm{Py}-\mathrm{H}) .-\mathrm{C}_{7} \mathrm{H}_{8} \mathrm{~N}_{2} \mathrm{O}_{3}$ : calcd. C 50.00, H 4.80, N 16.66; found C 49.59, H 5.02, N 16.72 .

\section{Procedure for the synthesis of compounds 15 and 16}

2-Phenylmalonaldehyde $(1.0 \mathrm{~g}, 6.75 \mathrm{mmol})$ and $\mathrm{N}$ carbamoyl L-valine $(1.62 \mathrm{~g}, 10.12 \mathrm{mmol})$ were mixed with toluene $(60 \mathrm{~mL})$. The mixture was refluxed with a Dean-Stark trap. Then it was cooled to $40{ }^{\circ} \mathrm{C}$, the toluene solution was decanted, and the resin residue was crystallized from ethanol.

(2S)-3-Methyl-2-((E)-3-(3-oxo-2-phenylpropylidene)ureido)butyric acid (15)

Colorless crystals. Yield 0.15 g (8\%); m.p. $203-204{ }^{\circ} \mathrm{C}$. $-{ }^{1} \mathrm{H}$ NMR (400 MHz, [D 6 DMSO): $\delta=0.85(\mathrm{~d}, J=6.8 \mathrm{~Hz}$, $\left.3 \mathrm{H}, \mathrm{CH}_{3}\right), 0.92$ (d, $\left.J=6.8 \mathrm{~Hz}, 3 \mathrm{H}, \mathrm{CH}_{3}\right), 2.04-2.21(\mathrm{~m}$,
$1 \mathrm{H}, \mathrm{CH}), 4.17-4.20(\mathrm{~m}, 1 \mathrm{H}, \mathrm{CH}), 7.21-7.23(\mathrm{~m}, 2 \mathrm{H}, \mathrm{Ph})$, $7.34-7.46(\mathrm{~m}, 4 \mathrm{H}, \mathrm{Ph}), 7.77(\mathrm{~d}, J=12.4 \mathrm{~Hz}, 1 \mathrm{H}), 9.14(\mathrm{~d}$, $J=12.4 \mathrm{~Hz}, 1 \mathrm{H}), 9.31$ (s, 1H, COOH), 12.63 (br.s, 1H, NH). - HRMS ((+)-ESI-MS): $m / z=291.1320$ (calcd. 291.1339 for $\left.\mathrm{C}_{15} \mathrm{H}_{19} \mathrm{~N}_{2} \mathrm{O}_{4},[\mathrm{M}+\mathrm{H}]^{+}\right)$.

1-Isobutyl-5-phenylpyrimidin-2(1H)-one (16)

Yellow crystals. Yield $0.20 \mathrm{~g}(13 \%) ;$ m.p. $177^{\circ} \mathrm{C} .-{ }^{1} \mathrm{H}$ NMR (400 MHz, [D 6 DMSO): $\delta=0.95(\mathrm{~d}, J=6.7 \mathrm{~Hz}, 6 \mathrm{H}$, $\left.2 \times \mathrm{CH}_{3}\right), 2.10-2.28(\mathrm{~m}, 1 \mathrm{H}, \mathrm{CH}), 3.77(\mathrm{~d}, J=7.4 \mathrm{~Hz}$, $\left.1 \mathrm{H}, \mathrm{CH}_{2}\right), 7.29-7.33(\mathrm{~m}, 1 \mathrm{H}, \mathrm{Ph}), 7.40-7.44(\mathrm{~m}, 2 \mathrm{H}, \mathrm{Ph})$, $7.55-7.57(\mathrm{~m}, 2 \mathrm{H}, \mathrm{Ph}), 8.48(\mathrm{~d}, J=3.4 \mathrm{~Hz}, 1 \mathrm{H}), 8.87(\mathrm{~d}$, $J=3.4 \mathrm{~Hz}, 1 \mathrm{H}) .-{ }^{13} \mathrm{C} \mathrm{NMR}\left(100 \mathrm{MHz},\left[\mathrm{D}_{6}\right] \mathrm{DMSO}\right): \delta=$ 164.8, 155.6, 147.4, 133.7, 129.5, 127.9, 125.8, 116.6, 57.9, 27.4, 19.9. - HRMS $((+)$-ESI-MS): $m / z=229.1352$ (calcd. 229.1335 for $\left.\mathrm{C}_{14} \mathrm{H}_{17} \mathrm{~N}_{2} \mathrm{O},[\mathrm{M}+\mathrm{H}]^{+}\right)$.

\section{Crystal structure determinations}

X-Ray analyses were carried out on an Oxford Diffraction Xcalibur S CCD diffractometer using the software package CRYSAlisPro [13]. X-Ray data collection was carried out at 295(2) K with graphite-monochromatized $\mathrm{Mo} K_{\alpha}$ radiation $(\lambda=0.71073 \AA)$. The structures were solved with Direct Methods using SHELXS-97 [14] and refined by full-matrix least-squares procedures on $F^{2}$ with SHELXL-97 [15]. Non$\mathrm{H}$ atoms were refined anisotropically, hydrogen atoms were placed in idealized positions and were constrained to ride on their parent atoms. Due to the absence of heavy atoms in the non-centrosymmetric crystal structures of 7 and 15, Friedel pairs were merged, and Flack parameters were not refined. Table 1 summarizes the crystal data and numbers pertinent to data collection and structure refinement.

CCDC 873468 (7), CCDC 936498 (15) and CCDC 936499 (16) contain the supplementary crystallographic data for this paper. These data can be obtained free of charge from The Cambridge Crystallographic Data Centre via www.ccdc. cam.ac.uk/data_request/cif.

\section{Acknowledgement}

We are pleased to thank Dr. P. Slepukhin for assistance with the X-ray analyses.
[1] T. Nishitani, T. Iwasaki, Y. Mushika, M. Miyoshi, J. Org. Chem. 1979, 44, 2019.

[2] G. E. Hardtmann, F. Park, H. Ott, US Pat. 3829422, 1974.

[3] P. Guy, H. Cousse, G. Mouzin, US Pat. 4677105, 1987.
[4] R. Berger, L. Chang, S. D. Edmondson, S. D. Goble, S. N. Ha, N. F. Kar, I. E. Kopka, B. Li, G. J. Morriello, C. R. Moyes, D.-M. Shen, L. Wang, C. Zhu, US Pat. 2009/0253705A1, 2009.

[5] R. Berger, L. Chang, S. D. Edmondson, S. D. Goble, B. Harper, N. F. Kar, I. E. Kopka, I. I. Bing, G. J. Mor- 
riello, C. R. Moyes, D.-M. Shen, L. Wang, H. Wendt, C. Zhu, WO Pat. 2009/123870A1, 2009.

[6] K. Papadopoulos, D. W. Young, Tetrahedron Lett. 2002, 43, 3951.

[7] P. B. Hitchcock, K. Papadopoulos, D. W. Young, Org. Biomol. Chem. 2003, 1, 2670.

[8] C. G. Overberger, J. Y. Chang, Tetrahedron Lett. 1989, 30,51 .

[9] V. Stella, T. Higuchi, J. Org. Chem. 1973, 38, 1527.

[10] E. Ware, Chem. Rev. 1950, 403.
[11] G. M. Coppola, G. E. Hardtmann, B. S. Huegi, J. Het. Chem. 1974, 11, 51

[12] V. F. Gonko, N. D. Shustova, G. M. Anoshina, T. E. Zubova, L. B. Radina, Pharm. Chem. J. 1978, 12, 601 (Engl. transl. from Khim.-Farm. Zh. 1978, 12, 53).

[13] Crysalis Pro, Oxford Diffraction Ltd., Abingdon, Oxford (U. K.) 2008.

[14] G. M. Sheldrick, Acta Crystallogr. 1990, A46, 467.

[15] G. M. Sheldrick, Acta Crystallogr. 2008, A64, 112. 